\title{
(2) OPEN ACCESS \\ Multiple primary malignancies: synchronous lymphoma, pancreatic neuroendocrine tumour and colorectal cancer
}

\author{
Nicolas Dayer, ${ }^{1}$ François Fasquelle, ${ }^{2}$ Emanuela Salati, ${ }^{3}$ Gilles Dietrich ${ }^{4}$
}

\begin{abstract}
'Internal Medicine, CHUV, Lausanne, Switzerland

${ }^{2}$ Pathology, CHUV, Lausanne, Switzerland

${ }^{3}$ Oncology, Hôpital Riviera-

Chablais, Rennaz, Switzerland

${ }^{4}$ Orthopedic Surgery, Hôpital

Riviera-Chablais, Rennaz,

Switzerland
\end{abstract}

Correspondence to

Dr Nicolas Dayer;

nico.dayer@hotmail.com

Accepted 12 May 2021

Check for updates

(c) BMJ Publishing Group Limited 2021. Re-use permitted under CC BY-NC. No commercial re-use. See rights and permissions. Published by BMJ.

To cite: Dayer $N$, Fasquelle $F_{\text {, }}$ Salati E, et al. BMJ Case Rep 2021;14:e241938. doi:10.1136/bcr-2021241938

\section{SUMMARY}

A 67-year-old woman underwent a medical checkup by her general practitioner after complaining of atypical pain in the shoulder girdle. Due to the important inflammatory syndrome noticed on blood testing, a polymyalgia rheumatica was suspected and she was started on corticosteroid treatment with good clinical response, but no impact on inflammation. She underwent extensive imaging with a thoraco-abdominal CT scanner that demonstrated a pancreatic mass, then later a PET-CT showed 3 different hyperactive lesions. Biopsies then revealed simultaneous diffuse large B-cell lymphoma (DLBCL), colorectal adenocarcinoma and pancreatic neuroendocrine tumour. She benefited from low rectal endoscopic excision of the colorectal tumour, R-CHOP chemotherapy for DLBCL and laparoscopic left pancreatectomy. Successful treatment required a good multidisciplinary collaboration between the different specialists. The patient made a good recovery and achieved complete remission at 1 year. This an unusual presentation of multiple primary malignancies.

\section{BACKGROUND}

Over the last several decades, the diagnosis of multiple primary malignancies (MPMs) has been established quite frequently. The incidence of this entity appears to be increasing due to multiple mechanisms implicated in its pathogenesis, including hereditary, immune and environmental factors such as chemicals, viruses, chemotherapeutic regimens and ionising radiation. ${ }^{12}$ In parallel, the increase in overall life expectancy, improved cancer therapies and more sensitive detection modalities ${ }^{2}$ have allowed the reported frequency of multiple synchronous primary tumours to increase to as high as $11.3 \%$ according to epidemiological studies. ${ }^{34}$ Common factors such as a strong smoking history, dietary factors, comorbidities, genetics and chronic inflammation may contribute to some extent to the development of synchronous malignancies as well. ${ }^{5}$

There is a high frequency of various neoplasia associated with carcinoid tumours, although the mechanisms behind these associations remain unclear. One hypothesis is that some of the bioactive substances secreted by these tumours are known mitogens for a variety of cell types and could therefore induce neoplastic transformation. ${ }^{6-8}$ Peptides such as secretin, gastrin, bombesin, cholecystokinin and VIP are believed to promote tumour cell growth. According to seven different studies, 4\%-26\% of carcinoid patients had a second malignancy. ${ }^{9}$ Of these lesions, approximately half $(53 \%)$ were adenocarcinoma of the gastrointestinal tract, of which $25 \%$ were colorectal carcinomas. ${ }^{10-12}$ In addition, Kamp et al reported that the incidence of synchronous malignancies was increased in patients with neuroendocrine tumour (NET) of the gastrointestinal tract and pancreas. ${ }^{13}$

The objective of this case report is to contribute to studies on the association of two or more tumours in multiple primary neoplasia, which could help to find new clues on the aetiology of cancers. Furthermore, as has been done by establishing appropriate and earlier surveillance of patients with carcinoid tumours, the management of patients with one tumour can also be improved if these studies identify those at highest risk of having a second tumour, thus allowing for more effective treatment of the second tumour. In the end, no consensus of opinion on guidelines for the treatment of MPMs was reached. This study aims to provide our experience on one case in this regard.

In the literature, several interesting cases of MPM involving different organs have been reported. However, to our knowledge, there is no case of synchronous primary triple malignancy involving colon, pancreas and a diffuse large B-cell lymphoma in a Mauritian woman.

\section{CASE PRESENTATION}

The patient was a 67-year-old Mauritian woman, otherwise in good health and without usual medication. During a visit to her general practitioner for a routine check-up, a significant inflammatory syndrome was found on blood tests with a high erythrocyte sedimentation sate $(>120 \mathrm{~mm} / \mathrm{h})$ and C reactive protein $(70-140 \mathrm{mg} / \mathrm{L})$ associated with an inflammatory anaemia $(\mathrm{Hb} 95 \mathrm{~g} / \mathrm{L})$. At this time, the patient complained of atypical pain in the shoulder girdle and a diagnosis of polymyalgia rheumatica (PMR) was suspected. Therefore, corticosteroid treatment was initiated. The joint symptoms disappeared, but the inflammatory syndrome remained fairly elevated. The patient had no B symptoms. She was a non-smoker and drank alcohol 2 to 3 times a month. There were no previous cancers in the family history.

\section{INVESTIGATIONS}

The in-depth evaluation was negative for the various factors of the PR. In this context, a thoracoabdominal CT scan was performed and showed a hypervascular body mass of the pancreas with 


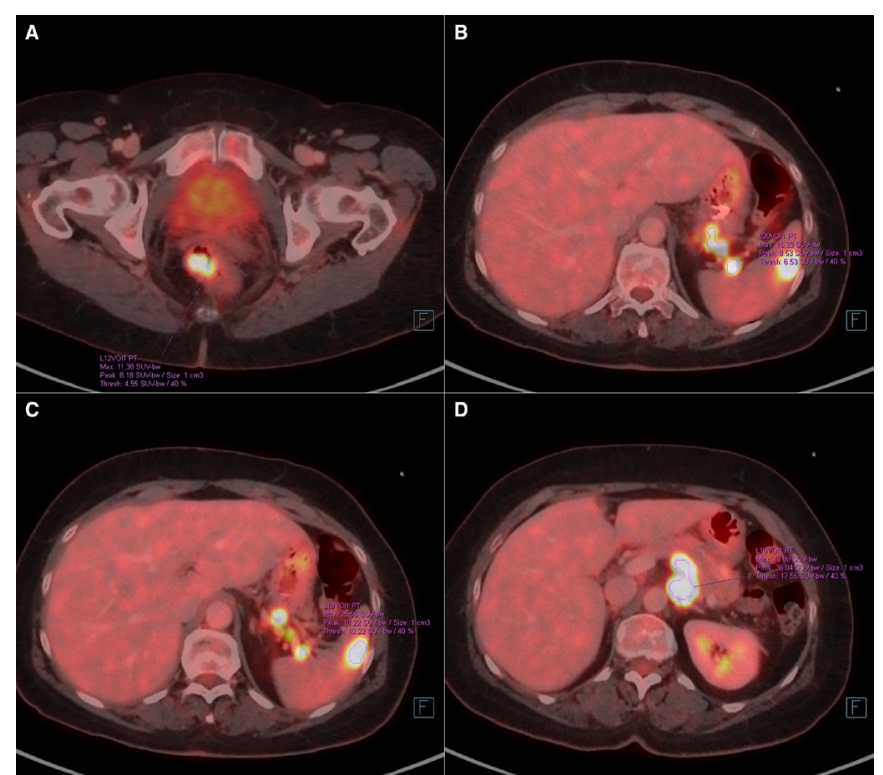

Figure 1 (A-D) PET-CT showing a metabolic uptake in a pancreatic body mass (standardised uptake value (SUV) max 4.3) (D), in the spleen (SUV max 25.5) (B, C), in multiple lymph nodes (mesentery root, small gastric curvature, splenic hila and retrocaval) (SUV max ranged from 5.7 to 48$)(B, C)$ and in the low rectum (SUV max 11.38) (A).

peri-pancreatic lymphadenopathies. A PET-CT scan performed for the staging revealed three different lesions based on uptake values. There was metabolic uptake in a pancreatic body mass (standardised uptake value (SUV) max 4.3), in the spleen (SUV max 25.5), in several lymph nodes (mesentery root, small gastric curvature, splenic hilum and retrocaval) (SUV max ranged from

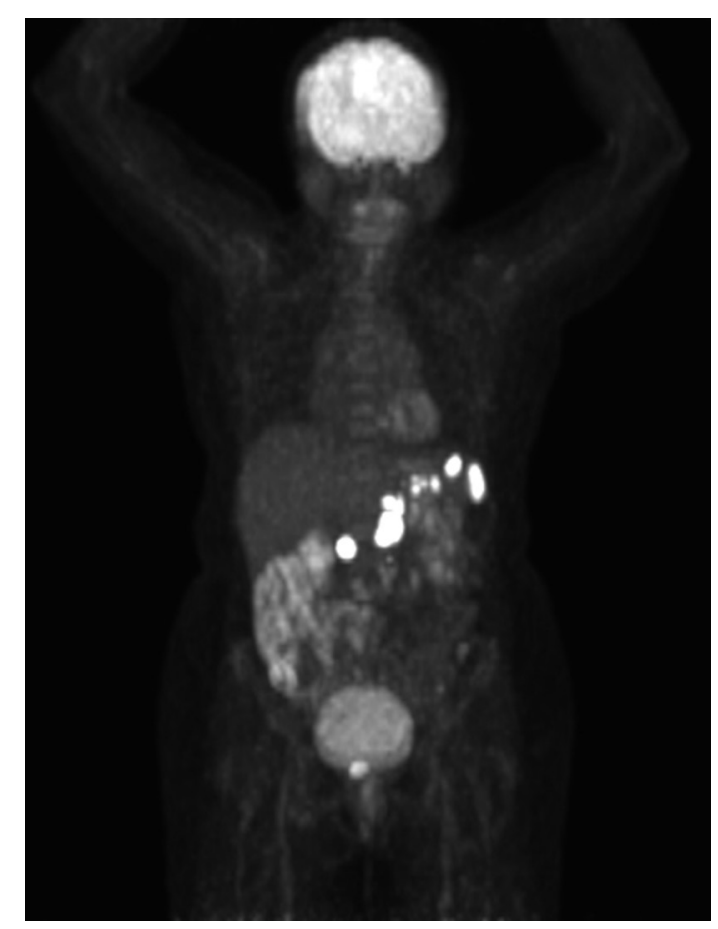

Figure 2 PET-CT showing a metabolic uptake in a pancreatic body mass (standardised uptake value (SUV) max 4.3), in the spleen (SUV max 25.5), in multiple lymph nodes (mesentery root, small gastric curvature, splenic hila and retrocaval) (SUV max ranged from 5.7 to 48) and in the low rectum (SUV max 11.38).
5.7 to 48 ) and in the low rectum (SUV max 11.38) (figures $1 \mathrm{~A}-\mathrm{D}$ and 2). A decision was made to perform several biopsies due to strong suspicion of distinct synchronous lesions.

Endoscopic evaluation of the pancreatic mass confirmed a $17 \times 17 \mathrm{~mm}$ pancreatic mass and biopsies confirmed that it was a well-differentiated NET on histopathology (pT2 pNx M0 L0 V0 Pn0 R0) (figure 3A-D). The same procedure resulted in the transgastrical biopsies by endosonography of a $23 \times 16 \mathrm{~mm}$ para-aortic ganglion for which histopathology revealed a nongerminal centre B-cell diffuse large B-cell lymphoma (non-GCB DLBCL) (figure 4A-D). During rectosigmoidoscopy, endoscopic resection of the $20 \mathrm{~mm}$ large rectal mass confirmed a moderately differentiated invasive adenocarcinoma (pT1 pNx L1 V0 Pn0 R1 G2) (figure 5). We did not perform a biopsy of the spleen lesion as it was close to the punctured para-aortic lymph node and having the same SUV max value, we deduced that it was probably the same lesion. The puncture was also omitted to avoid significant bleeding from the spleen.

The three tumours were morphologically and immunohistochemically different. Biopsy of the NET of the pancreas harboured acinar and solid islets, poorly proliferative and immunoreactive for pancytokeratin, synaptophysin and chromogranin. PAX8 was also expressed, consistent with a welldifferentiated neuroendocrine tumour (NET-G1) of pancreatic origin. CDX2 and TTF1 immunostains were negative.

The para-aortic lymph node core needle biopsy disclosed a diffuse large lymphoid cell proliferation. Immunohistochemistry revealed these cells to be highly proliferative and of B type, expressing CD20 but not CD3, CD5, CD10, CD23 or Bcl-1. Immunostains for Bcl-6, MUM1 were also positive. Molecular analysis (PCR and FISH) revealed an IgH clonal rearrangement and a Bcl- 6 translocation. The diagnosis of DLBCL was made of non-GCB type according to Hans.

Histological analysis of the rectal tumour resected endoscopically in two pieces revealed an atypical glandular proliferation of a moderately differentiated adenocarcinoma, invading submucosa, with lymphatic invasion.

The mismatch repair status was stable on both the pancreatic and the rectal tumour, with a preserved expression of the



Figure 3 (A-D) Pathological findings of pancreatic tumour. (A) H\&E stain, magnification factor $\times 4$. (B) Chromogranin A immunohistochemistry positive, magnification factor $\times 20$. (C) H\&E stain, magnification factor $\times 20$. (D) Synaptophysin immunohistochemistry positive, magnification factor $\times 20$. 


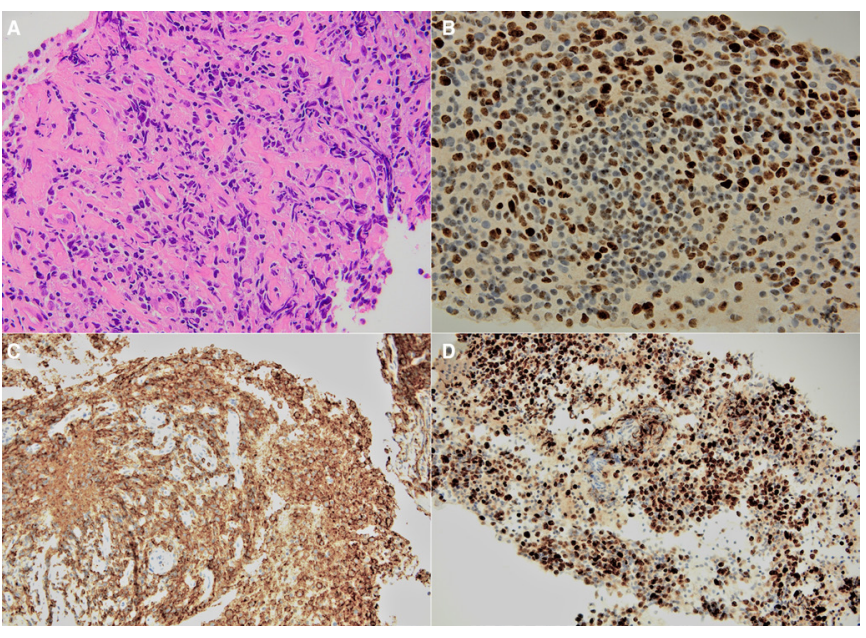

Figure 4 (A-D) Pathological findings of para-aortic biopsy diagnostic for diffuse large B-cell lymphoma. (A) H\&E, magnification factor $\times 20$. (B) CD20 immunohistochemistry (IHC) positive, magnification factor $\times 20$.

(C) Bcl6 IHC positive, magnification factor $\times 10$. (D) MIB IHC revealing a high proliferation index, magnification factor $\times 10$.

MLH1, MSH2, MSH6 and PMS2 proteins. There was therefore no argument in favour of a Lynch syndrome.

To ensure that there was no common origin between the rectal and pancreatic tumour, a comparative molecular analysis by next-generation sequencing (NGS, a panel of 52 gene hotspot mutations commonly found in solid malignant neoplasms) was performed. It revealed a TP53, KRAS and APC mutation as well as a SMAD4 deletion in the rectal adenocarcinoma and did not reveal any mutation in the NGS gene panel in pancreatic NET, concluding in the absence of genomic link between the two tumours.

\section{TREATMENT}

A multidisciplinary team of internists, oncologists, gastroenterologists and surgeons proposed a combination therapy plan. The decision was made to treat first the lymphoma as the most aggressive tumour. The patient was treated with 6 cycles of R-CHOP chemotherapy (rituximab, cyclophosphamide, doxorubicin, vincristine and prednisolone) for high-grade B lymphoma and achieved a complete response to treatment.

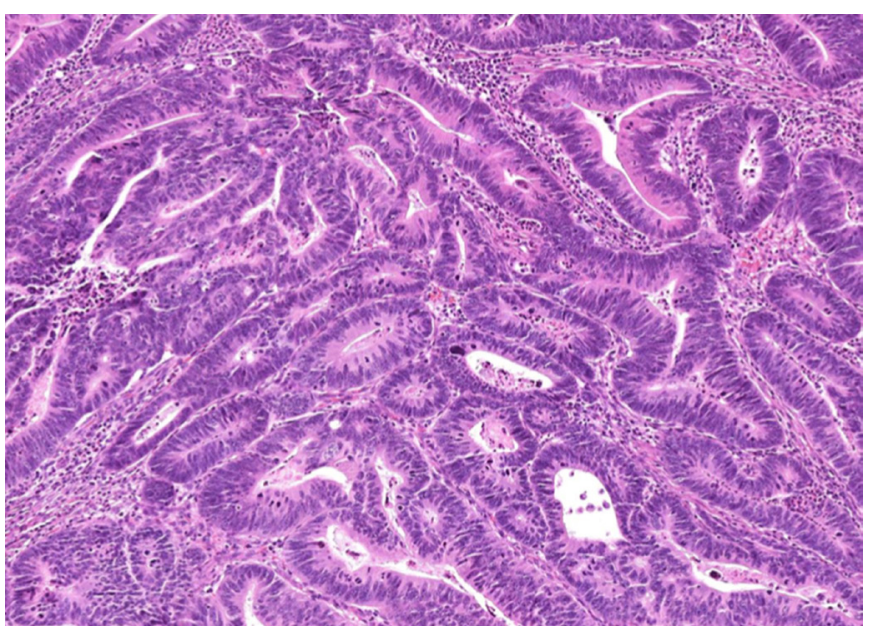

Figure 5 Pathological findings of rectal tumour. H\&E stain, magnification factor $\times 20$.

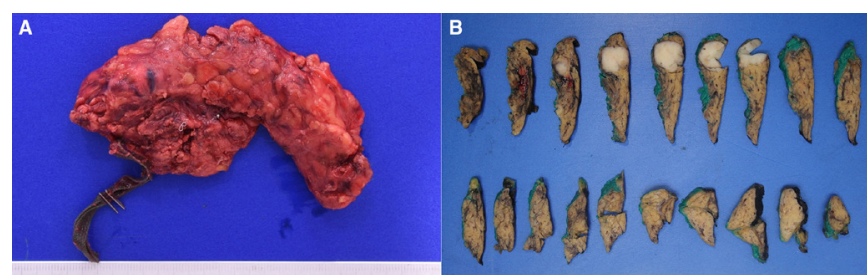

Figure 6 (A, B) Macroscopical findings of the left pancreatectomy surgical specimen.

The low rectal adenocarcinoma was excised by colonoscopy at time of diagnosis.

A PET-CT scan performed during follow-up showed no evidence of recurrence of either lymphoma or rectal cancer. With the lymphoma cured, it was then decided to treat the remaining tumour with laparoscopy to remove the left part of the pancreas for the neuroendocrine pancreatic tumour, with an attempt to preserve the spleen and the splenic vessels (figure 6A,B).

\section{OUTCOME AND FOLLOW-UP}

The removal of the rectal polyp was initially for biopsy purposes and was done before chemotherapy. A follow-up colonoscopy with biopsy samples was performed 2 months later and did not reveal any residual tumour cells. The follow-up by MRI performed at 7 and 12 months and recto-sigmoidoscopy performed at 8 months post-excision did not show any recurrence of rectal malignancy. The tumour was then considered cured.

The resection of the pancreatic tumour was successful with complete remission. The patient was still in remission at the 1-year follow-up scan.

\section{DISCUSSION}

Three criteria have been established by Warren and Gates to characterise MPMs: (I) each tumour must be distinct from the other; (II) each must have well-defined malignancy characteristics; (III) the possibility that one is a metastatic lesion derived from the other must be excluded. ${ }^{14-16}$

The term of MPMs commonly includes two large categories depending on the time of diagnosis of each tumour. Synchronous neoplasms are defined as such if they are diagnosed simultaneously or within 6 months. If a second or high order malignancies follow one another at least 6 months after the previous one, they are called metachronous. ${ }^{14-17}$ We can therefore state that our patient suffered from an MPM consisting of three synchronous neoplasms.

Despite its increasing rates, MPMs remain rare, as mentioned previously. Population-based studies have attempted to estimate the incidence of a second or third primary malignancy in an individual who has already been diagnosed with cancer. Schoenberg et al found that patients with cancer were 1.29 times more likely to develop a new malignancy than patients without cancer. ${ }^{17}$ The risk is slightly higher for women to develop metachronous tumours but in the other hand synchronous lesions slightly favour men. ${ }^{2}$

As seen in the introduction, the study of MPMs can provide clues about the aetiology and management of cancer. These entities can generally be separated into three main groups based on the main aetiological factor. The first group includes neoplasms related to cancer treatment, the second includes syndromic cases and the third includes neoplasms that may share common aetiological factors, such as genetic predisposition or the same environmental factors. ${ }^{18}$ In addition, two or more cancers may also be the result of pure chance. ${ }^{19}$ 
Our patient had no risk factors for cancer and showed no familial predisposition. Unlike some tumours that share the same embryological origin, such as urological and gynaecological tumours for example, our patient's three tumours had different embryological origins. Furthermore, no known syndrome or genetic predisposition was identified by our analyses and comparative molecular tests of the pancreatic and rectal tumours disclosed no common mutation, so we believe that the three tumours result from a sporadic occurrence.

MPMs, when discovered, raise questions about possible common aetiological factors and pathogenetic mechanisms, and more importantly, they cause many problems for clinicians and patients as treatment options generally become limited.

Treatment protocols for MPM are not well established. As the combination of our patient's three tumours had never been described before, we could not rely on a previously reported treatment protocol. Thus, as the prognosis depends on the lymphoma, which is the most aggressive neoplasm, the choice of therapy for the three cancers followed an order of severity in terms of time and was considered separately for each one in terms of efficacy. Thus, histopathological confirmation is crucial in these patients for appropriate treatment recommendations. Based on our experience, the detection of three limited stage primaries allowed us to establish a customised treatment plan with a good early outcome.

The lymphoma was treated with R-CHOP chemotherapy, the most appropriate treatment for this pathology. Subsequently, the tumour of the pancreas was treated surgically (left pancreatectomy), and the least severe tumour was removed directly at the time of diagnosis (endoscopic resection). Due to the mucosal resection and biopsy showing invasion of submucosa with lymphatic invasion, we discussed the need of an adjuvant therapy to prevent recurrence of rectal cancer. The multidisciplinary team (MDT) decided to control by close follow-up with colonoscopy, biopsies and imageries. This approach proved to be effective in this case. Moreover, the treatment of our patient's tumours resulted in the disappearance of the initial symptoms that she was complaining about and the discontinuation of the corticosteroids she was on. Indeed, we remind you that our patient initially had PMR symptoms that responded to the corticosteroids, but the markers of inflammation remained elevated. Naschitz highlighted the atypical features of the PMR suspected of underlying malignancy: age $<50$ years; limited or asymmetrical involvement of typical sites; an erythrocyte sedimentation rate $<40$ or $>100 \mathrm{~mm} / \mathrm{h}$; poor or incomplete response to low doses of corticosteroids; long-lasting symptoms. Malignancies reported were in decreasing order of frequency, myeloproliferative and myelodysplastic syndromes followed by breast, colon, kidney and prostate cancers. ${ }^{20}$

Rheumatological disorders such as rheumatic polymyalgia, inflammatory myopathies, seronegative arthritis and atypical vasculitis have been associated with the clinical manifestations of certain solid and haematological tumours. They may even precede the development of cancers and contribute to the early diagnosis and effective treatment of these conditions. It has been demonstrated by Racanelli et al that medical treatment or surgical removal of tumours can lead to a regression in the clinical manifestations of these paraneoplastic rheumatological disorders. ${ }^{21}$

Difficulties encountered in the management of this case were due to the atypical presentation and diagnosis of three malignant lesions with different histopathology. The rapid evocation of a differential diagnosis in the face of this atypical presentation and the aggressive diagnostic approach of a MDT can help overcome these challenges. There are no standard guidelines for the management of this type of MPM, but the type of malignancies, disease course, response to therapy and the general condition of the patients should always be taken into consideration. If the lesions are curable, clinicians should opt for radical therapy as observed in our patient.

\section{Learning Points}

- This is an unusual presentation of multiple primary malignancies of colon adenocarcinoma, pancreatic neuroendocrine tumour and lymphoma. It seems to be diagnosed with a higher incidence than that predicted by the influence of hazard alone.

- The study of this case may provide useful information regarding the development of effective screening and monitoring protocols in order to treat patients with similar conditions effectively.

- A multidisciplinary team approach with effective teamwork and specialist knowledge and skills is necessary to approach and treat these complex cases.

- Patients with synchronous primaries can be treated aggressively depending on the stage of the individual tumour.

- We must also keep in mind that atypical course of rheumatological disorders should draw attention to aspects that may reveal hidden tumours.

Contributors ND was the internist in charge of the patient in the medical ward, FF was the pathologist in charge of analysing the different biopsies and specimens, ES was the oncologist in charge of the chemotherapeutical treatment and the follow-up GD was in the surgical team. Redaction and conceptualisation of the case report was led by ND and GD.

Funding The authors have not declared a specific grant for this research from any funding agency in the public, commercial or not-for-profit sectors.

Competing interests None declared.

Patient consent for publication Obtained.

Provenance and peer review Not commissioned; externally peer reviewed.

Open access This is an open access article distributed in accordance with the Creative Commons Attribution Non Commercial (CC BY-NC 4.0) license, which permits others to distribute, remix, adapt, build upon this work non-commercially, and license their derivative works on different terms, provided the original work is properly cited and the use is non-commercial. See: http://creativecommons.org/ licenses/by-nc/4.0/.

\section{REFERENCES}

1 Warren $\mathrm{S}$, Gates $\mathrm{O}$. Multiple primary malignant tumors: survey of the literature and a statistical study. Am J Cancer 1932;16:1358-414.

2 Spratt JS, Hoag MG. Incidence of multiple primary cancers per man-year of follow up: 20-year review from the Ellis Fischel State Cancer Hospital. Ann Surg 1966;164:775-84.

3 Copur MS, Manapuram S. Multiple primary tumors over a lifetime. Oncology 2019;33:629384

4 Demandante CGN, Troyer DA, Miles TP. Multiple primary malignant neoplasms: case report and a comprehensive review of the literature. Am J Clin Oncol 2003;26:79-83.

5 Puri A, Ma L, Walker GV, et al. Synchronous primary adenocarcinoma of the lung and pancreas: a case series and review of the literature. Lung Cancer Manag 2017;6:17-23

6 ÖBERG K. Expression of growth factors and their receptors in neuroendocrine gut and pancreatic tumors, and prognostic factors for survival. Ann N Y Acad Sci 1994:733:46-55

7 Modlin IM, Lye KD, Kidd M. A 5-decade analysis of 13,715 carcinoid tumors. Cancer 2003:97:934-59.

8 Modlin IM, Goldenring JR, Lawton GP, et al. Aspects of the theoretical basis and clinical relevance of low acid states. Am J Gastroenterol 1994;89:308-18.

9 Verrico M, Rossi L, Tomao S, et al. Metachronous and synchronous cancers in patients with neuroendocrine tumors. Oncology 2020;98:10-15.

10 Marshall JB, Bodnarchuk G. Carcinoid tumors of the gut. our experience over three decades and review of the literature. J Clin Gastroenterol 1993;16:123-9. 
11 Clift AK, Drymousis P, Al-Nahhas A, et al. Incidence of second primary malignancies in patients with neuroendocrine tumours. Neuroendocrinology 2015;102:26-32.

12 Olney JR, Urdaneta LF, Al-Jurf AS, et al. Carcinoid tumors of the gastrointestinal tract. Am Surg 1985:51:37-41.

13 Kamp K, Damhuis RAM, Feelders RA, et al. Occurrence of second primary malignancies in patients with neuroendocrine tumors of the digestive tract and pancreas. Endocr Relat Cancer 2012;19:95-9.

14 Sakellakis M, Peroukides S, Iconomou G, et al. Multiple primary malignancies: a report of two cases. Chin J Cancer Res 2014;26:215-8.

15 Aydiner A, Karadeniz A, Uygun K, et al. Multiple primary neoplasms at a single institution. Am J Clin Oncol 2000;23:364-70.

16 Derwinger K, Gustavsson B. A study of aspects on gender and prognosis in synchronous colorectal cancer. Clin Med Insights Oncol 2011;5:CMO.S7871.
17 Schoenberg BS. Multiple primary malignant neoplasms. The Connecticut experience, 1935-1964. Recent Results Cancer Res 1977:58:1-173.

18 Takalkar U, Asegaonkar BN, Kodlikeri P, et al. An elderly woman with triple primary metachronous malignancy: a case report and review of literature. Int I Surg Case Rep 2013;4:593-6.

19 Wood ME, Vogel V, Ng A, et al. Second malignant neoplasms: assessment and strategies for risk reduction. J Clin Oncol 2012;30:3734-45.

20 Naschitz JE. Rheumatic syndromes: clues to occult neoplasia. Curr Opin Rheumatol 2001;13:62-6.

21 Racanelli V, Prete M, Minoia C, et al. Rheumatic disorders as paraneoplastic syndromes. Autoimmun Rev 2008;7:352-8.

Copyright 2021 BMJ Publishing Group. All rights reserved. For permission to reuse any of this content visit

https://www.bmj.com/company/products-services/rights-and-licensing/permissions/

BMJ Case Report Fellows may re-use this article for personal use and teaching without any further permission.

Become a Fellow of BMJ Case Reports today and you can:

- Submit as many cases as you like

- Enjoy fast sympathetic peer review and rapid publication of accepted articles

- Access all the published articles

Re-use any of the published material for personal use and teaching without further permission

\section{Customer Service}

If you have any further queries about your subscription, please contact our customer services team on +44 (0) 2071111105 or via email at support@bmj.com.

Visit casereports.bmj.com for more articles like this and to become a Fellow 